\title{
Prevalence of vitamin K deficiency and associated factors in non-supplemented cystic fibrosis patients
}

\author{
Patrycja Krzyżanowska, Ph.D., ${ }^{a}$ Stawomira Drzymała-Czyż, Ph.D., ${ }^{a}$ \\ Nataliya Rohovyk, MSc, Lyudmyla Bober, Ph.D., ${ }^{b} J e r z y ~ M o c z k o$, Prof., ${ }^{c}$ \\ Marta Rachel, Ph.D., ${ }^{d}$ and Jarostaw Walkowiak, Prof. ${ }^{a}$
}

a. Department of Pediatric Gastroenterology and Metabolic Diseases, Poznan University of Medical Sciences, Poznan, Poland.

b. Lviv Cystic Fibrosis Centre, Lviv, Ukraine.

c. Department of Informatics and Statistics, Poznan University of Medical Sciences, Poznan, Poland.

d. Allergology Outpatient Department, Provincial Hospital No 2, Rzeszow, Poland.

E-mail address:

Prof. Jarosław

Walkowiak:

jarwalk@ump.edu.pl

Funding:

Supported by the Ministry of Sciences and Higher Education (grant No. N N407 531238).

Conflict of interest:

None.

Received: 12-22-2016

Accepted: 8-15-2017

\begin{abstract}
Introduction. Vitamin $\mathrm{K}$ deficiency is highly prevalent in cystic fibrosis (CF) patients despite supplementation. Moreover, no reliable risk factors for its occurrence are known. The aim was to assess the prevalence of vitamin K deficiency and associated factors in non-supplemented CF patients.

Methods. Prothrombin concentration induced by vitamin $\mathrm{K}$ absence (PIVKA-II) and the undercarboxylated osteocalcin percentage (u-OC) were determined. In all patients clinical status was assessed and its relation to vitamin $\mathrm{K}$ deficiency determined. The following tests were used for statistical analysis: Mann-Whitney test, ANOVA test or the Kruskal Wallis test, the chi-squared test or the Fisher-Freeman-Halton test, and multiple linear and multiple forward stepwise logistic regression analysis.

Results. The study group comprised $79 \mathrm{CF}$ patients aged 0.4-25.3 years. PIVKA-II and u-OC were abnormal in $56(70.9 \%)$ and $45(57.0 \%)$ patients. Patients with elevated PIVKA-II were significantly older $(p=0.0184)$ and had lower Z-score values for body weight ( $p=0.0297$ ) than those with normal concentrations. Patients with normal or pathological u-OC percentage did not differ. Abnormal PIVKA-II and u-OC were reported more frequently in subjects with two severe CFTR mutations and with worse/poor nutritional status. Multiple linear and forward stepwise regression analyses did not reveal strong predictive factors of vitamin Kdeficiency. Conclusion. Vitamin K deficiency is highly prevalent in the natural course of cystic fibrosis. There are no reliable clinical determinants of its occurrence.

Key words: PIVKA-II (prothrombin induced by vitamin $K$ absence), undercarboxylated osteocalcin, gastrointestinal diseases, child, adolescent.
\end{abstract}

http:/ / dx.doi.org/10.5546/ aap.2018.eng.e19

To cite: Krzyżanowska P, Drzymała S, Rohovyk N, et al. Prevalence of vitamin $\mathrm{K}$ deficiency and associated factors in non-supplemented cystic fibrosis patients. Arch Argent Pediatr 2018;116(1):e19-e25.

\section{GLOSSARY}

ALT: alanine transaminase ANOVA: analysis of variance AST: aspartate transaminase Body height (Z-score): standardized value of body height Body weight (Z-score): standardized value of body weight CF: cystic fibrosis

CFTR: cystic fibrosis transmembrane conductance regulator

FEV1: forced expiratory volume in

1 second

GGT: gamma-glutamyl transferase

$\mathrm{K} 1$ : vitamin K1 (phylloquinone)

$\mathrm{K} 2$ : vitamin $\mathrm{K} 2$ (menaquinone)

MK: menaquinone

PCR: polymerase chain reaction

PIVKA-II: prothrombin induced by vitamin $\mathrm{K}$ absence

PT: prothrombin time

RFLP: restriction fragment length polymorphism

u-OC: undercarboxylated osteocalcin.

\section{INTRODUCTION}

Vitamin $\mathrm{K}$, a fat-soluble vitamin, exists in two natural forms as vitamin K1 (phyloquinone) and $\mathrm{K} 2$ (menaquinone). Vitamin $\mathrm{K} 1$ is found in large amounts in green leafy vegetables and vegetable oil. The primary source of vitamin K2 is fermented food..$^{1-3}$ Moreover, longchain menaquinones, such as MK-7, MK-8, MK-9, MK-10, MK-11 and MK12 are synthesized by colonic flora (Bacteroides). ${ }^{3}$

Vitamin $\mathrm{K}$ is essential for gammacarboxylation of vitamin K-dependent proteins, such as coagulation factors (II, VII, IX, X, protein S and C), osteocalcin, matrix-Gla protein, kidney Gla protein and growth-arrest- 
specific-6 protein. ${ }^{4-6}$ Data indicates that vitamin K plays several biological roles. In addition to its participation in blood coagulation, vitamin $\mathrm{K}$ is important for bone mineralization, the regulation of vascular calcification, cancer prevention and improved insulin sensitivity., ${ }^{4-10}$

Vitamin K deficiency in patients with cystic fibrosis (CF) is thought to be caused by intestinal malabsorption due to pancreatic insufficiency and bile salt deficiency, liver disease, bowel resection, antibiotic treatments and inadequate dietary intake. ${ }^{11,12}$ Vitamin K deficiency predisposes CF patients to easy bruising and bleeding (especially among infants), defective bone mineralization and osteoporosis. ${ }^{13-15}$

Routine vitamin K supplementation in CF patients is recommended in many countries. ${ }^{14}$ However, vitamin $\mathrm{K}$ deficiency is frequently reported in CF subjects despite supplementation. ${ }^{16-21}$ Therefore, identifying potential risk factors for vitamin $\mathrm{K}$ deficiency in CF patients seems to be very important. Nevertheless, reliable predictive factors have not been determined for $\mathrm{CF}$ subjects receiving vitamin $\mathrm{K} .{ }^{21}$ Hence, the aim of the present study was to assess the prevalence of vitamin $\mathrm{K}$ deficiency and associated factors in nonsupplemented cystic fibrosis patients.

\section{POPULATION AND METHODS}

Participants were recruited from December 2010 to December 2012. All patients who were treated in Lviv Cystic Fibrosis Centre were selected in routine follow-up visits. The main inclusion criteria were $\mathrm{CF}$ diagnosis and written consent for the study. Individuals were excluded if they had serious medical conditions (endstage pulmonary disease with EV1 $<20 \%$ ) or if they had received vitamin $\mathrm{K}$ supplementation. CF diagnosis was based on generally accepted guidelines..$^{22}$ Data on participants were collected on a regular basis throughout the study.

Nutritional status (standardized body height and weight, albumin concentration ${ }^{23}$ ), clinical expression of disease (lung function, spirometry, exocrine pancreatic function, fecal elastase- $1,24-26$ Pseudomonas aeruginosa colonization, and biochemical markers of liver damage - ALT, AST, $\mathrm{GGT}^{27}, \mathrm{PT}^{28}$ ) were assessed at the time of blood sample collection in all CF patients. Additionally, information about coexisting diabetes and liver cirrhosis was collected. Pseudomonas aeruginosa colonization refers to isolation of the bacteria from the sputum at least once within a 6-month period prior to the study. The most common CFTR gene mutations in Western Ukraine were identified earlier in both alleles to confirm the cystic fibrosis diagnosis using polymerase chain reaction (PCR) and heteroduplex analysis or RFLP (Restriction Fragment Length Polymorphism).

Vitamin K status was estimated by measuring both the concentration of prothrombin induced by vitamin $\mathrm{K}$ absence (PIVKA-II) and the percentage of undercarboxylated osteocalcin (u-OC) using immunoassay kits (DeCarboxy Prothrombin, Diagnostica Stago, Asnières-sur-Seine, France; Glatype Osteocalcin EIA Takara BIO INC. Otsu, Shiga, Japan; Glu-type Osteocalcin EIA Takara BIO INC. Otsu, Shiga, Japan) as described earlier. ${ }^{21}$

The differences between the subgroups with normal (PIVKA-II $<2 \mathrm{ng} / \mathrm{ml}$ ) and abnormal (PIVKA-II $\geq 2 \mathrm{ng} / \mathrm{ml}$ ) vitamin $\mathrm{K}$ status were assessed using the Mann-Whitney test. In turn, differences between subgroups of patients with normal (u-OC $<20 \%$ ), insufficient (u-OC $20-50 \%$ ) and deficient ( $\mathrm{u}-\mathrm{OC}>50 \%$ ) vitamin $\mathrm{K}$ status were assessed using the ANOVA test (for Gaussian distribution) or the Kruskal Wallis test (for non-Gaussian distribution). The Shapiro-Wilk test was applied to determine the normality of data distribution. The frequency of vitamin $\mathrm{K}$ deficiency was estimated using the chi-squared test (for PIVKA-II) or the Fisher-FreemanHalton test (for u-OC). Multiple linear regression analysis and multiple forward stepwise logistic regression analysis were used to estimate the potential influence of all studied parameters on the occurrence of vitamin $\mathrm{K}$ deficiency in two models with different distributions of CFTR gene mutations. All independent variables were included in the regression models. The decision on including or excluding predictors which were statistically not significant was taken by analyzing partial and semi-partial correlation coefficients and by examining the collinearity of predictors using the tolerance coefficient. The level of significance was set at $p<0.05$. Statistical analyses were carried out using StatSoft. Inc (2014) STATISTICA (data analysis software system version 12 ).

The study was conducted in accordance with the revised Declaration of Helsinki. Informed written consent was obtained from adult patients who were 16 years old or older or from patients' parents when the patients were younger than 16 . The study was approved by the Bioethical Committee at Poznan University of Medical Sciences (Resolution No 640/2009) and the Ethical 
Committee of the Western Ukrainian Specialized Children's Medical Centre in Lviv (Resolution No 9a/2012).

\section{RESULTS}

One hundred available CF patients were screened. Three $\mathrm{CF}$ patients were excluded because they had FEV1 under 20\%. Four subjects received vitamin K supplementation, and 14 subjects enrolled at the preliminary stage dropped out

TABLE 1. Clinical parameters of study patients $(N=79)$

\begin{tabular}{lc}
\hline Clinical parameters & Median $\left(\mathbf{1}^{\text {st }-3^{\text {rd }}}\right.$ quartile $)$ \\
\hline Age [years] & $8.5(5.1-13.6)$ \\
Body weight (Z-score) & $-1.1(-1.5--0.7)$ \\
Body height (Z-score) & $-1.0(-1.9--0.2)$ \\
Albumin [g/L] & $39.0(37.0-41.0)$ \\
FEV1 [\%] & $77.0(58.5-88.0)$ \\
GGT [U/L] & $14.7(13.0-16.8)$ \\
ALT [U/L] & $22.0(17.5-33.5)$ \\
AST [U/L] & $31.0(24.0-37.5)$ \\
PT [s] & $16.2(15.1-17.4)$ \\
\hline
\end{tabular}

FEV1: forced expiratory volume in 1 second,

GGT: gamma-glutamyl transferase, ALT: alanine transaminase, AST: aspartate transaminase, PT: prothrombin time,

${ }^{1} \mathrm{FEV} 1$ was assessed in 59 patients. Age of the participants (below 6 years old) determined the possibility of performing the test. of the study due to the loss of blood samples in transport. Finally, 79 CF patients aged 0.4-25.3 years (35 [44.3\%] females and 44 [55.7\%] males) who have never received vitamin $\mathrm{K}$ were included to the study group. Clinical, biochemical and functional characteristics of the studied patients are summarized in Table 1.

Five $(6.3 \%)$ patients were pancreatic sufficient, while the remaining $74(93.7 \%)$ subjects presented steatorrhea. Sixty-five $(82.3 \%)$ patients were colonized with Pseudomonas aeruginosa. Only one $(1.3 \%)$ CF patient had diabetes and $5(6.3 \%)$ patients had liver cirrhosis.

Mutations in one or both alleles of CFTR gene were identified in all patients - CFTR gene mutations in bold refer to severe mutations. The genotypes of the studied patients were as follows: F508del/F508del $(n=26), F 508 \mathrm{del} /-(\mathrm{n}=11)$, F508del/2184insA $(\mathrm{n}=9)$, F508del/N1303K $(\mathrm{n}=7), \mathbf{F} 508 \mathrm{del} / \mathbf{1 8 9 8}+\mathbf{1 G}-\mathbf{A}(\mathrm{n}=4), \mathbf{F} 508 \mathrm{del} /$ G542X $(\mathrm{n}=4)$, F508del/CFTR dele2,3(21kb) $(\mathrm{n}=3)$, F508del / 3849+10kbC/T ( $=2)$, F508del/ W1282X $(n=2)$, F508del / 185+1G $>T(n=1)$, F508del/2143delT ( $=1$ ), F508del/621-1G >T $(n=1)$, F508del / R347H ( $n=1)$, F508del/R553X $(\mathrm{n}=1)$, 2184insA/2184insA $(\mathrm{n}=1)$, 2184insA/ N1303K $(n=1), 3272-11 A>G / 3272-11 A>G(n=1)$,

TABLE 2. Clinical, biochemical and functional parameters in CF patients with normal and pathological PIVKA-II concentration $(N=79)$

\begin{tabular}{|c|c|c|c|c|c|c|c|c|}
\hline \multicolumn{9}{|c|}{ PIVKA-II [ng/ml] } \\
\hline $\begin{array}{l}\text { Parameters } \\
\text { Median } \\
\left(1^{\text {st }}-3^{\text {rd }} \text { quartile }\right)\end{array}$ & $\begin{array}{c}<2 \\
N=23\end{array}$ & $\begin{array}{c}\geq 2 \\
\mathrm{~N}=56\end{array}$ & $p$ & & ters & $\begin{array}{c}<2 \\
N=23\end{array}$ & $\begin{array}{c}\geq 2 \\
N=56\end{array}$ & $p$ \\
\hline \multirow{2}{*}{ Age [years] } & \multirow{2}{*}{$\begin{array}{c}7.8 \\
(4.3-12.2)\end{array}$} & \multirow{2}{*}{$\begin{array}{c}11.4 \\
(7.8-14.8)\end{array}$} & \multirow{2}{*}{0.0184} & \multirow{2}{*}{$\begin{array}{l}\text { Body weight } \\
\text { (Z-score) }\end{array}$} & $<-1$ & 6 & 28 & \multirow{2}{*}{0.0512} \\
\hline & & & & & $>-1$ & 17 & 28 & \\
\hline \multirow{2}{*}{$\begin{array}{l}\text { Body weight } \\
\text { (Z-score) }\end{array}$} & \multirow{2}{*}{$\begin{array}{c}-1.0 \\
(-1.4--0.6)\end{array}$} & \multirow{2}{*}{$\begin{array}{c}-1.3 \\
(-1.9--1.0)\end{array}$} & \multirow[b]{2}{*}{0.0297} & \multirow{2}{*}{$\begin{array}{l}\text { Body height } \\
\text { (Z-score) }\end{array}$} & $<-1$ & 8 & 31 & \multirow{2}{*}{0.0966} \\
\hline & & & & & $>-1$ & 15 & 25 & \\
\hline \multirow{2}{*}{$\begin{array}{l}\text { Body height } \\
\text { (Z-score) }\end{array}$} & \multirow{2}{*}{$\begin{array}{c}-0.8 \\
(-1.7--0.1)\end{array}$} & \multirow{2}{*}{$\begin{array}{c}-1.4 \\
(-2.5--0.4)\end{array}$} & \multirow{2}{*}{0.1422} & \multirow{2}{*}{ Albumin [g/l] } & $<35$ & 3 & 4 & \multirow{2}{*}{0.6872} \\
\hline & & & & & $\geq 35$ & 20 & 52 & \\
\hline \multirow{2}{*}{ Albumin $[\mathrm{g} / \mathrm{dl}]$} & \multirow{2}{*}{$\begin{array}{c}39.0 \\
(36.4-41.3)\end{array}$} & \multirow{2}{*}{$\begin{array}{c}39.0 \\
(37.5-41.0)\end{array}$} & \multirow{2}{*}{0.7578} & \multirow{2}{*}{ FEV1 [\%] } & $<80 \%$ & 6 & 18 & \multirow{2}{*}{0.1593} \\
\hline & & & & & $>80 \%$ & 15 & 20 & \\
\hline \multirow{2}{*}{ FEV1 [\%] } & \multirow{2}{*}{$\begin{array}{c}79.0 \\
(59.9-90.3)\end{array}$} & \multirow{2}{*}{$\begin{array}{c}68.0 \\
(58.0-81.0)\end{array}$} & \multirow{2}{*}{0.1732} & \multirow{2}{*}{$\begin{array}{l}\text { Pancreatic } \\
\text { sufficiency }\end{array}$} & Yes & 2 & 3 & \multirow{2}{*}{0.9641} \\
\hline & & & & & No & 21 & 53 & \\
\hline \multirow{2}{*}{ PT [s] } & \multirow{2}{*}{$\begin{array}{c}16.5 \\
(15.6-17.3) \\
\end{array}$} & \multirow{2}{*}{$\begin{array}{c}16.0 \\
(15.1-17.3)\end{array}$} & & Ps. aeruginosa & Yes & 18 & 47 & \\
\hline & & & 0.5524 & colonization & No & 5 & 9 & 0.5490 \\
\hline ALT [U/L] & 24.0 & 22.0 & 05855 & liver cirrbosis & Yes & 0 & 5 & 03310 \\
\hline & $(18.0-33.0)$ & $(17.0-33.5)$ & 0.5855 & Liver cirrnosis & No & 23 & 51 & 0.3310 \\
\hline AST [U/L] & 34.0 & 30.5 & & Diabetec & Yes & 0 & 1 & 06436 \\
\hline & $(22.0-35.5)$ & $(24.0-38.5)$ & 0.9012 & Dlabetes & No & 23 & 55 & 0.6436 \\
\hline & 140 & 149 & & & F508del/F508del & 5 & 21 & 01756 \\
\hline GGT [U/L] & 14.0 & 14.9 & & CFTR gene & other/other & 18 & 35 & 0.1750 \\
\hline ] & $(118-173)$ & $(13,0-16.5)$ & 0.4038 & mutations & severe/severe & 15 & 48 & \\
\hline & $(11.0-11.0)$ & $(13.0-10.5)$ & & & other/other & 8 & 8 & 0.0395 \\
\hline
\end{tabular}

PIVKA-II: prothrombin induced by vitamin K absence, FEV1: forced expiratory volume in 1 second, PT: prothrombin time, ALT: alanine transaminase, AST: aspartate transaminase, GGT: gamma-glutamyl transferase,

CFTR: cystic fibrosis transmembrane conductance regulator.

${ }^{1}$ FEV1 was assessed in 59 patients. Age of the participants (below 6 years old) determined the possibility of perform the test. 
621-1G > T / 3849+10kbC $>$ T $(\mathrm{n}=1), \mathrm{G} 542 X / N 1303 K$ $(\mathrm{n}=1), \mathbf{N 1 3 0 3 K} / 2183 A A-G(\mathrm{n}=1)$.

Pathological PIVKA-II concentrations and abnormal percentage of $\mathrm{u}-\mathrm{OC}$ were found in $56(70.9 \%)$ and $45(57.0 \%)$ CF patients who were not receiving vitamin $\mathrm{K}$.

The study subgroups with normal and pathological PIVKA-II concentrations differed significantly in terms of age $(p=0.0184)$ and standardized body weight ( $p=0.0297)$. However, patients with normal and pathological vitamin $\mathrm{K}$ status based on the percentage of $\mathrm{u}-\mathrm{OC}$ did not differ significantly (Table 2 and Table 3 ).

Vitamin K deficiency based on PIVKA-II and $\mathrm{u}-\mathrm{OC}$ was more frequently reported in $\mathrm{CF}$ subjects with two severe mutations in both alleles of CFTR gene and with standardized body weight below one standard deviation (Table 2 and Table 3 ).

According to the multiple linear regression analysis in models defined in relation to F508del/other and severe/other, GGT was potentially defined as a determinant of PIVKA-II concentration (Table 4). The regression model for $\mathrm{u}-\mathrm{OC}$ was not statistically significant.

Based on the multiple forward stepwise logistic regression analysis, GGT, AST, FEV1 and albumin concentration (in both regression models) together explained $58.3 \%$ of PIVKA-II concentration variance (Table 5).

\section{DISCUSSION}

In the present study we found a high prevalence of vitamin $\mathrm{K}$ deficiency in CF patients. It was more frequent in subjects with two severe CFTR gene mutations and with worse nutritional status. Moreover, PIVKA-II concentration was significantly dependent on GGT activity. This may suggest that GGT activity could be a strong predictor of vitamin $\mathrm{K}$ status. However, in most patients, GGT activity was within the reference range. Therefore, this finding - although statistically significant - does not seem to have any clinical importance.

Considering the biological role of vitamin $\mathrm{K}$, its supplementation seems to be very important in CF patients who are at risk of its deficiency. Moreover, it is necessary to carry out studies which could explain the existence of vitamin $\mathrm{K}$ deficiency in CF patients despite its supplementation. ${ }^{16-21}$ Therefore, finding endogenous and exogenous determinants of vitamin $\mathrm{K}$ deficiency could help to determine the appropriate vitamin $\mathrm{K}$ dosage in order to maintain its normal body resources in CF patients. This study is the first to recruit a large group of CF patients without vitamin $\mathrm{K}$ supplementation in order to analyze potential determinants of its deficiency. It is worth emphasizing that vitamin $\mathrm{K}$ supplementation in CF patients is mandatory

TABLE 3. Clinical, biochemical and functional parameters in CF patients with normal and pathological u-OC percentage $(N=79)$.

\begin{tabular}{|c|c|c|c|c|c|c|c|c|c|c|}
\hline \multicolumn{11}{|c|}{$\mathrm{u}-\mathrm{OC}[\%]$} \\
\hline $\begin{array}{l}\text { Parameters } \\
\text { Median } \\
\left(4^{\text {st }}-3^{\text {rd }}\right. \\
\text { quartile })\end{array}$ & $\begin{array}{c}<20 \\
(\mathrm{~N}=34)\end{array}$ & $\begin{array}{l}20-50 \\
(N=29)\end{array}$ & $\begin{array}{c}\geq 50 \\
(\mathrm{~N}=16)\end{array}$ & $p$ & \multicolumn{2}{|c|}{$\begin{array}{l}\text { Parameters } \\
\text { (N) }\end{array}$} & $\begin{array}{c}<20 \\
(\mathrm{~N}=34)\end{array}$ & $\begin{array}{c}20-50 \\
(\mathrm{~N}=29)\end{array}$ & $\begin{array}{c}\geq 50 \\
(\mathrm{~N}=16)\end{array}$ & $p$ \\
\hline \multirow{2}{*}{ Age [years] } & \multirow{2}{*}{$\begin{array}{c}9.9 \\
(5.0-14.3)\end{array}$} & \multirow{2}{*}{$\begin{array}{c}7.8 \\
(5.1-12.1)\end{array}$} & \multirow{2}{*}{$\begin{array}{c}9.2 \\
(5.7-11.9) \\
\end{array}$} & \multirow{2}{*}{0.9631} & \multirow{2}{*}{$\begin{array}{l}\text { Body weight } \\
\text { (Z-score) }\end{array}$} & $<-1$ & 14 & 20 & 11 & \multirow{2}{*}{0.0480} \\
\hline & & & & & & $>-1$ & 20 & 9 & 5 & \\
\hline \multirow{2}{*}{$\begin{array}{l}\text { Body weight } \\
\text { (Z-score) }\end{array}$} & \multirow{2}{*}{$\begin{array}{c}-0.9 \\
(-1.5--0.5)\end{array}$} & \multirow{2}{*}{$\begin{array}{c}-1.1 \\
(-1.4--0.9)\end{array}$} & \multirow{2}{*}{$\begin{array}{c}-1.3 \\
(-1.8--0.9)\end{array}$} & \multirow{2}{*}{0.1630} & \multirow{2}{*}{$\begin{array}{l}\text { Body height } \\
\text { (Z-score) }\end{array}$} & $<-1$ & 16 & 16 & 8 & \multirow{2}{*}{0.8105} \\
\hline & & & & & & $>-1$ & 18 & 13 & 8 & \\
\hline \multirow{2}{*}{$\begin{array}{l}\text { Body height } \\
\text { (Z-score) }\end{array}$} & \multirow{2}{*}{$\begin{array}{c}-0.9 \\
(-1.7--0.2)\end{array}$} & \multirow{2}{*}{$\begin{array}{c}-1.1 \\
(-2.3--0.5)\end{array}$} & \multirow{2}{*}{$\begin{array}{c}-1.1 \\
(-2.4--0.0)\end{array}$} & \multirow{2}{*}{0.8962} & \multirow{2}{*}{ Albumin [g/l] } & $<35$ & 3 & 4 & 0 & \multirow{2}{*}{0.4023} \\
\hline & & & & & & $\geq 35$ & 31 & 25 & 16 & \\
\hline \multirow{2}{*}{ Albumin $[\mathrm{g} / \mathrm{dl}]$} & \multirow{2}{*}{$\begin{array}{c}40.5 \\
(38.1-43.0)\end{array}$} & \multirow{2}{*}{$\begin{array}{c}39.0 \\
(37.0-40.0)\end{array}$} & \multirow{2}{*}{$\begin{array}{c}39.5 \\
(36.4-41.7)\end{array}$} & \multirow{2}{*}{0.2655} & \multirow{2}{*}{ FEV1 [\%] } & $<80 \%$ & 13 & 14 & 8 & \multirow{2}{*}{0.5915} \\
\hline & & & & & & $>80 \%$ & 12 & 7 & 5 & \\
\hline \multirow{2}{*}{ FEV1 [\%] } & \multirow{2}{*}{$\begin{array}{c}79.9 \\
(65.0-88.0)\end{array}$} & \multirow{2}{*}{$\begin{array}{c}70.0 \\
(49.0-88.0)\end{array}$} & & & Pancreatic & Yes & 1 & 2 & 2 & \\
\hline & & & $(61.4-85.0)$ & 0.9098 & sufficiency & No & 33 & 27 & 14 & 0.3415 \\
\hline & 16.0 & 16.3 & 16.5 & & Ps. aeruginosa & Yes & 30 & 24 & 11 & \\
\hline $\mathrm{Pl}[\mathrm{s}]$ & $(15.2-17.1)$ & $(15.0-17.5)$ & $(15.4-17.2)$ & 0.9662 & colonization & No & 4 & 5 & 5 & 0.2417 \\
\hline & 22.5 & 22.0 & 26.0 & & & Yes & 2 & 3 & 0 & \\
\hline ALT [U/L] & $(17.0-30.0)$ & $(19.0-29.0)$ & $(20.0-37.3)$ & 0.5350 & Liver cirrhosis & No & 32 & 26 & 16 & 0.6073 \\
\hline AST [U/L] & 28.5 & 35.0 & 33.5 & & & Yes & 1 & 0 & 0 & \\
\hline & $(23.3-35.0)$ & $(26.0-40.0)$ & $(24.0-38.5)$ & $0.26 / 9$ & Diabetes & No & 33 & 29 & 16 & $>0.9999$ \\
\hline & & & & & & F508del/F508del & 13 & 11 & 2 & \\
\hline GGT [U/L] & 14.0 & 15.0 & 14.9 & 08775 & CFTR gene & other/other & 21 & 18 & 14 & 0.1503 \\
\hline & $(13.0-17.0)$ & $(13.7-16.0)$ & $(13.0-17.6)$ & 0.8175 & mutation & severe/severe & 30 & 23 & 10 & \\
\hline & & & & & & other/other & 4 & 6 & 6 & 0.1069 \\
\hline
\end{tabular}

u-OC: undercarboxylated osteocalcin, FEV1: forced expiratory volume in 1 second, PT: prothrombin time,

ALT: alanine transaminase, AST: aspartate transaminase, GGT: gamma-glutamyl transferase,

CFTR: cystic fibrosis transmembrane conductance regulator.

${ }^{1} \mathrm{FEV} 1$ was assessed in 59 patients. Age of the participants (below 6 years old) determined the possibility of perform the test. 
in most countries. Therefore, recruiting such a large, homogenous group of CF patients is not easy. Only CF subjects who had never received vitamin $\mathrm{K}$ were included, which is the main methodological advantage of the present study. The present study is an exploratory study without calculation of sample size, which constitutes its disadvantage. The limitation of the present study

TABLE 4. Multiple linear regression analysis $(N=55)$

\begin{tabular}{|c|c|c|}
\hline Clinical parameters & $\begin{array}{l}\text { First model }^{1}(\mathrm{~N}=55)^{3} \\
\text { PIVKA-II }[\mathrm{ng} / \mathrm{ml}]\end{array}$ & $\begin{array}{l}\text { Second model }^{2}(\mathrm{~N}=55)^{3} \\
\text { PIVKA-II }[\mathrm{ng} / \mathrm{ml}]\end{array}$ \\
\hline$p$ model & 0.00036 & 0.00037 \\
\hline $\mathrm{R}^{\wedge} 2$ for model & 0.59611957 & 0.59607034 \\
\hline Adjusted R2 for model & 0.44078094 & 0.44071278 \\
\hline Age & $0.852330\{-1.120 \pm 5.9753\}^{4}$ & $0.848371\{-1.148 \pm 5.9622\}$ \\
\hline Gender & $0.948066\{3.283 \pm 50.0720\}$ & $0.953523\{2.921 \pm 49.8039\}$ \\
\hline Body weight (Z-score) & $0.458950\{-45.349 \pm 60.6275\}$ & $0.458970\{-46.029 \pm 61.5391\}$ \\
\hline Body height (Z-score) & $0.723354\{12.968 \pm 36.3711\}$ & $0.719490\{13.245 \pm 36.6145\}$ \\
\hline Albumin $[\mathrm{g} / \mathrm{L}]$ & $0.495537\{-4.058 \pm 5.8987\}$ & $0.518373\{-3.830 \pm 5.8759\}$ \\
\hline FEV1 $[\%]$ & $0.195707\{1.497 \pm 1.1368\}$ & $0.207399\{1.511 \pm 1.1790\}$ \\
\hline $\mathrm{PT}[\mathrm{s}]$ & $0.709356\{-4.834 \pm 12.8757\}$ & $0.698906\{-5.124 \pm 13.1486\}$ \\
\hline GGT $[\mathrm{U} / \mathrm{L}]$ & $0.000941\{9.868 \pm 2.7570\}$ & $0.001153\{9.853 \pm 2.8084\}$ \\
\hline ALT $[\mathrm{U} / \mathrm{L}]$ & $0.780313\{0.812 \pm 2.8917\}$ & $0.782390\{0.822 \pm 2.9559\}$ \\
\hline AST $[\mathrm{U} / \mathrm{L}]$ & $0.465931\{3.051 \pm 4.1435\}$ & $0.455000\{3.096 \pm 4.1021\}$ \\
\hline Diabetes & $0.912137\{-20.484 \pm 184.4372\}$ & $0.900524\{-24.792 \pm 197.0522\}$ \\
\hline Liver cirrhosis & $0.733231\{-40.866 \pm 119.0469\}$ & $0.755672\{-38.720 \pm 123.5607\}$ \\
\hline Pancreatic insufficiency / sufficiency & $0.553867\{57.521 \pm 96.3284\}$ & $0.573268\{54.703 \pm 96.3021\}$ \\
\hline Pseudomonas aeruginosa colonization & $0.988139\{1.186 \pm 79.2976\}$ & $0.981113\{1.930 \pm 80.9913\}$ \\
\hline CFTR gene mutation & $0.936988\{-4.263 \pm 53.5715\}$ & $0.968525\{2.639 \pm 66.4497\}$ \\
\hline
\end{tabular}

PIVKA-II: prothrombin induced by vitamin K absence, FEV1: forced expiratory volume in 1 second, PT: prothrombin time, GGT: gamma-glutamyl transferase, ALT: alanine transaminase, AST: aspartate transaminase,

CFTR: cystic fibrosis transmembrane conductance regulator.

$R^{\wedge} 2$ : adjusted $R 2$, regression slope coefficient \pm standard error of regression slope coefficient were presented only for statistically significant model.

${ }^{1}$ CFTR gene mutations were divided as follows: F508del/F508del, other/other.

${ }^{2}$ CFTR gene mutations were divided as follows: severe/severe, other/other.

${ }^{3}$ Multiple linear regression analysis was estimated in 55 patients because 24 participants did not have complete data (in 20 subjects a lack of FEV1 and in 4 a lack of GGT activity).

TABLE 5. Multiple forward stepwise logistic regression analysis $(N=55)$

\begin{tabular}{|c|c|c|c|c|c|c|}
\hline$p$ model & $R^{\wedge} 2$ & Adjusted R2 for model & Dependent variable & Independent variable & $\mathrm{b} \pm$ standard error $^{4}$ & $p$ \\
\hline \multicolumn{7}{|c|}{ First model $^{1}(\mathrm{~N}=55)^{3}$} \\
\hline$<0.00000$ & 0.58337112 & 0.55004081 & PIVKA-II [ng/ml] & $\begin{array}{c}\text { GGT [U/L] } \\
\text { AST [U/L] } \\
\text { FEV1 [\%] } \\
\text { Albumin [g/L] }\end{array}$ & $\begin{array}{c}8.727 \pm 1.8992 \\
4.091 \pm 2.2035 \\
0.998 \pm 0.7793 \\
-4.538 \pm 4.4977 \\
\end{array}$ & $\begin{array}{l}0.000030 \\
0.069267 \\
0.206195 \\
0.317808 \\
\end{array}$ \\
\hline \multicolumn{7}{|c|}{ Second model $^{2}(\mathrm{~N}=55)^{3}$} \\
\hline$<0.00000$ & 0.58337112 & 0.55004081 & PIVKA-II [ng/ml] & $\begin{array}{c}\text { GGT [U/L] } \\
\text { AST [U/L] } \\
\text { FEV1 [\%] } \\
\text { Albumin [g/L] }\end{array}$ & $\begin{array}{c}8.727 \pm 1.8992 \\
4.091 \pm 2.2035 \\
0.998 \pm 0.7793 \\
-4.538 \pm 4.4977\end{array}$ & $\begin{array}{l}0.000030 \\
0.069267 \\
0.206195 \\
0.317808\end{array}$ \\
\hline
\end{tabular}

PIVKA-II: prothrombin induced by vitamin K absence, FEV1: forced expiratory volume in 1 second, AST: aspartate transaminase, GGT: gamma-glutamyl transferase.

$R^{\wedge} 2$, adjusted $R 2$, regression slope coefficient \pm standard error of regression slope coefficient were presented only for statistically significant model.

${ }^{1}$ CFTR gene mutations were divided as follows: F508del/F508del, other/other.

${ }^{2}$ CFTR gene mutations were divided as follows: severe/ severe, other/other.

${ }^{3}$ Multiple linear regression analysis was estimated in 55 patients because 24 participants did not have complete data (in 20 subjects a lack of FEV1 and in 4 a lack of GGT activity).

${ }^{4}$ regression slope coefficient \pm standard error of regression slope coefficient. 
is an indirect measurement of vitamin $\mathrm{K}$ using PIVKA-II concentration and u-OC percentage. On the other hand, however, PIVKA-II and u-OC reflect the biological role of vitamin $\mathrm{K}$.

Available evidence indicates some risk factors associated with decreased vitamin $\mathrm{K}$ in both supplemented and non-supplemented CF patients. It has been suggested that exocrine pancreatic insufficiency and malabsorption of fats and bile salts in CF patients can lead to fat-soluble vitamin deficiency, including vitamin K. ${ }^{14,29,30}$ Rashid et al., found significantly higher levels of PIVKA-II in CF patients with pancreatic insufficiency without vitamin K supplementation $(n=83)$ compared with subjects with normal pancreatic function $(\mathrm{n}=15) .{ }^{31}$ Nicolaidou et al., found significant differences in u-OC (median 4.0 vs. $8.1 \mathrm{ng} / \mathrm{ml}, p=0.017)$ and c-OC concentrations (median 22.0 vs. $13.8 \mathrm{ng} / \mathrm{ml}, p=0.002$ ) among healthy individuals $(n=25)$ and CF patients with pancreatic insufficiency without vitamin $\mathrm{K}$ supplementation $(n=20) .{ }^{32}$ Similarly, Hoorn et al., documented significantly higher PIVKAII concentrations in pancreatic insufficient CF patients who were not receiving vitamin $\mathrm{K}$ $(\mathrm{n}=10)$ or were receiving a low dose $(<0.25 \mathrm{mg} /$ day) $(n=6)$ compared with healthy subjects $(\mathrm{n}=9) .{ }^{33}$

Liver disease is also listed as one of the risk factors of vitamin K deficiency in CF patients. ${ }^{14}$ Mosler et al., demonstrated normal PIVKAII concentrations only in two cases among 15 CF subjects with liver disease who were receiving oral ursodeoxycholic acid due to high GGT activity. ${ }^{13}$ Rashid et al., and Wilson et al., documented pathological PIVKA-II concentrations in all CF patients with liver disease ( $n=8$ and $n=6$, respectively). ${ }^{31,34}$ Recently, we found evidence that vitamin $\mathrm{K}$ deficiency is more frequent in CF patients with liver cirrhosis than in those without. However, liver cirrhosis was not documented to be an independent risk factor of vitamin K deficiency in CF. ${ }^{35} \mathrm{In}$ the past, long-term use of antibiotics was also considered a potential risk factor of vitamin K deficiency because it could decrease vitamin $\mathrm{K}$ production by colonic flora ${ }^{17}$. However, Beker et al. and Rashid et al., did not find significant correlation between antibiotic treatment and PIVKA-II concentrations in CF patients. ${ }^{16,31}$

In our another recent study, ${ }^{21}$ we documented that pathological concentrations of PIVKA-II occurred more frequently in CF patients with pancreatic insufficiency and those who have two severe mutations in both alleles of the CFTR gene. The pathological percentage of $\mathrm{u}-\mathrm{OC}$ was found more frequently in adult CF patients and those who were not receiving vitamin K. ${ }^{21}$ Multiple linear regression analysis showed that none of the independent variables (age, Z-score for body weight and height, FEV1, albumin concentration, vitamin $\mathrm{K}$ dose $\mathrm{mg} /$ week, diabetes, liver disease, pancreatic insufficiency, Pseudomonas aeruginosa colonization, inhaled and oral permanent antibiotic therapy, intravenous and oral antibiotic therapy in the preceding three months, inhaled glucocorticoids therapy, CFTR mutation) were important for predicting PIVKA-II or u-OC status. However, based on multiple stepwise logistic regression analysis, we demonstrated that liver disease, diabetes and glucocorticoid therapy were potential determinants of PIVKA-II concentrations and that the dose of vitamin $\mathrm{K}$ was a potential determinant of $\mathrm{u}$-OC percentage in CF patients. ${ }^{21}$ Dougherty et al., also presented multiple linear regression models predicting vitamin $\mathrm{K}$ status in supplemented CF subjects, which were separately represented as $\mathrm{u}$-OC percentages for females and males. They documented that vitamin $\mathrm{K}$ supplementation, $25(\mathrm{OH}) \mathrm{D}_{3^{\prime}}$ age for males, and vitamin $\mathrm{K}$ supplementation only for females were significant predictors of $\mathrm{u}$-OC percentage. ${ }^{19}$

In conclusion, vitamin $\mathrm{K}$ deficiency is highly prevalent in the natural course of cystic fibrosis. There are no reliable clinical determinants of its occurrence.

\section{REFERENCES}

1. McCann JC, Ames BN. Vitamin K, an example of triage theory: is a micronutrient inadequacy linked to diseases of aging? Am J Clin Nutr. 2009;90(4):889-907.

2. Schurgers LJ, Vermeer C. Determination of phylloquinone and menaquinones in food. Effect of food matrix on circulating vitamin $\mathrm{K}$ concentrations. Haemostasis. 2000;30(6):298-307.

3. Shearer MJ, Newman P. Metabolism and cell biology of vitamin K. Thromb Haemost. 2008;100(4):530-47.

4. Booth SL. Roles for Vitamin K beyond coagulation. Annu Rev Nutr. 2009;29:89-110.

5. Greer FR. Vitamin K the basics - What's new? Early Hum Dev. 2010;86 (Suppl 1):S43-7.

6. Cranenburg EC, Schurgers LJ, Vermeer C. Vitamin K: the coagulation vitamin that became omnipotent. Thromb Haemost. 2007;98(1):120-5.

7. Wu FY, Liao WC, Chang HM. Comparison of antitumor activity of vitamins $\mathrm{K} 1, \mathrm{~K} 2$ and $\mathrm{K} 3$ on human tumor cells by two (MTT and SRB) cell viability assays. Life Sci. 1993;52(22):1797-804.

8. Koshihara $Y$, Hoshi K. Vitamin K2 enhances osteocalcin accumulation in the extracellular matrix of human osteoblasts in vitro. J Bone Miner Res. 1997;12(3):431-8. 
9. Shiraki $\mathrm{M}$, Shiraki $\mathrm{Y}$, Aoki $\mathrm{Ch}$, et al. Vitamin $\mathrm{K} 2$ (Menatetrenone) effectively prevents fractures and sustains lumbar bone mineral density in osteoporosis. J Bone Miner Res. 2000;15(3):515-21.

10. DiNicolantonioJJ, BhutaniJ, O'KeefeJH. The Heath benefits of vitamin K. Open Heart. 2015;2(1):e000300.

11. Conway SP. Vitamin Kin cystic fibrosis. J R Soc Med. 2004;97 Suppl 44:48-51.

12. Krzyżanowska $P$, Walkowiak J. Vitamin K status in cystic fibrosis patients. Acta Sci Pol Technol Aliment. 2010;9(4): 463-7.

13. Mosler K, von Kries R, Vermeer C, et al. Assessment of vitamin $\mathrm{K}$ deficiency in $\mathrm{CF}$ - how much sophistication is useful? J Cyst Fibros. 2003;2(2):91-6.

14. Jagannath VA, Fedorowicz Z, Thaker V, et al. Vitamin K supplementation for cystic fibrosis. Cochrane Database Syst Rev. 2015;1:CD008482.

15. Cottam ST, Connett GJ. Routine use of daily oral vitamin $\mathrm{K}$ to treat infants with cystic fibrosis. Pediatr Respir Rev. 2015;165 Suppl 1:22-4.

16. Beker LT, Ahrens RA, Fink RJ, et al. Effect of vitamin K1 supplementation on vitamin $\mathrm{K}$ status in cystic fibrosis patients. J Pediatr Gastroenterol Nutr. 1997;24(5):512-7.

17. Conway SP, WolfeSP, Brownlee KG, et al. Vitamin Kstatus among children with cystic fibrosis and its relationship to bone mineral density and bone turnover. Pediatrics. 2005;115(5):1325-31.

18. Drury D, Grey VL, Ferland G, et al. Efficacy of high dose phylloquinone in correcting vitamin $\mathrm{K}$ deficiency in cystic fibrosis. J Cyst Fibros. 2008;7(5):457-9.

19. Dougherty KA, Schall JI, Stallings VA. Suboptimal vitamin $\mathrm{K}$ status despite supplementation in children and young adults with cystic fibrosis. Am J Clin Nutr. 2010;92(3):660-7.

20. Krzyżanowska P, Lisowska A, Woś $\mathrm{H}$, et al. Vitamin $\mathrm{K}$ status in young children with cystic fibrosis. Acta Sci Pol Technol Aliment. 2011;10(3):399-406.

21. Krzyżanowska P, Pogorzelski A, Skorupa W, et al. Exogenous and endogenous determinants of witamin $\mathrm{K}$ status in cystic fibrosis. Sci Rep. 2015;5:12000.

22. Castellani C, Southern KW, BrownleeK, et al.European best practice guidelines for cystic fibrosis neonatal screening. $J$ Cyst Fibros. 2009;8(3):153-73.
23. Doumas BT, Watson WA, Biggs HG. Albumin standards and the measurement of serum albumin with bromcresol green. Clin Chim Acta. 1971;31(1):87-96.

24. Walkowiak J, Nousia-Arvanitakis S, Cade A, et al. Fecal elastase-1 cut-off levels in the assessment of exocrine pancreatic function in cystic fibrosis. J Cyst Fibros. 2002;1(4):260-4.

25. Walkowiak J, Lisowska A, Przyslawski J, et al. Faecal elastase- 1 test is superior to faecal lipase test in the assessment of exocrine pancreatic function in cystic fibrosis. Acta Pediatr. 2004;93(8):1042-5.

26. Walkowiak J, Lisowska A, Błaszczyński M. The changing face of the exocrine pancreas in cystic fibrosis: pancreatic sufficiency, pancreatitis and genotype. Eur J Gastroenterol Hepatol. 2008;20(3):157-60.

27. Schumann G, Bonora R, Ceriotti $F$, et al. IFCC primary reference procedures for the measurement of catalyticactivity concentrations of enzymes at 37 degrees C. International Federation of Clinical Chemistry and Laboratory Medicine. Clin Chem Lab Med. 2002;40(7):718-38.

28. QuickAJ. The thromboplastin reagent for the determination of prothrombin. Science. 1940;92(2379):113-4.

29. Borgo G, Mastella G, Gasparini P, et al. Pancreatic function and gene deletion F508 in cystic fibrosis. J Med Genet. 1990;27(11):665-9.

30. Maqbool A, Stallings VA. Update on fat-soluble vitamins in cystic fibrosis. Curr Opin Pulm Med. 2008;14(6):574-81.

31. Rashid M, Durie $P$, Andrew M, et al. Prevalence of vitamin K deficiency in cystic fibrosis. Am J Clin Nutr. 1999;70(3): 378-82.

32. Nicolaidou P, Stavrinadis I, Loukou I, et al. The effect of vitamin Ksupplementation on biochemical markers of bone formation in children and adolescents with cystic fibrosis. Eur J Pediatr. 2006;165(8):540-5.

33. van Hoorn JH, Hendriks JJE, Vermeer C, et al. Vitamin $\mathrm{K}$ supplementation in cystic fibrosis. Arch Dis Child. 2003;88(11):974-5.

34. Wilson DC, Rashid M, Durie PR, et al. Treatment of vitamin $\mathrm{K}$ deficiency in cystic fibrosis: Effectiveness of a daily fatsoluble vitamin combination. J Pediatr. 2001;138(6):851-5.

35. Krzyżanowska P, Drzymała-Czyż S, Pogorzelski A, et al. Vitamin K status in cystic fibrosis patients with liver cirrhosis. Dig Liver Dis. 2017;49(6):672-5. 